\title{
Transjugular intrahepatic portosystemic shunt related paradoxical air embolism during orthotopic liver transplantation
}

\author{
Douglas M. Kinscherff, MD • Paul Picton, FRCA • \\ Joshua Kollars, MD · Pema Dorje, MD
}

Received: 17 May 2009/Accepted: 2 November 2009/Published online: 30 December 2009

(C) Canadian Anesthesiologists' Society 2009

\section{To the Editor,}

During the dissection phase of an orthotopic liver transplant, a 63 yr old man developed widespread ST depression and cardiovascular collapse. Transesophageal echocardiography (TEE) revealed air in both ventricles associated with new onset biventricular dilation and dysfunction. The surgeons found a failed staple line placed inadvertently across a transjugular intrahepatic portosystemic shunt (TIPS), which was now splinting the right hepatic vein open. Control of air entrainment was achieved with caval clamping. Trendelenberg positioning was employed to prevent air entering the right ventricular outflow tract. Following resuscitation, a full echocardiographic examination was undertaken. Air was seen extending into the aortic root but was not demonstrated in the coronary circulation. Patent foramen ovale (PFO) was not demonstrated on colour Doppler. A plausible explanation for this paradoxical air embolism is intrapulmonary shunting. Intrapulmonary shunt recruitment is thought to occur by a pressure dependent baffle mechanism ${ }^{1}$ exacerbated in this case by increased pulmonary artery pressures from air embolus.

Potentially catastrophic hemodynamic changes occur frequently during orthotopic liver transplantation (OLT) and require rapid and accurate diagnosis. During the dissection phase, hepatic manipulation causes intermittent obstruction to venous return. The anhepatic phase involves clamping the portal vein and inferior vena cava, both of which cause a

D. M. Kinscherff, MD $(\varangle) \cdot$ P. Picton, FRCA

J. Kollars, MD · P. Dorje, MD

University of Michigan, Ann Arbor, USA

e-mail: dkinsche@med.umich.edu significant reduction in venous return. Reperfusion syndrome ( $>30 \%$ decrease in mean arterial pressure for at least $1 \mathrm{~min}$ ) is seen in $42 \%$ of patients. $^{2}$ Associated physiologic changes include increased pulmonary pressures, right ventricular dysfunction, bradycardia, acidosis, and hypothermia. Given the multiple causes of hypotension and hemodynamic instability during OLT, the assessment of cardiac preload and function is easily justified. ${ }^{3}$

In comparison with a pulmonary artery catheter, TEE has the distinct advantage of being a relatively non-invasive procedure. TEE allows direct visualization of the heart, permitting monitoring of volume status, contractility, and overall function. In addition, TEE provides valuable information when less common complications occur, such as large pleural effusions, tension pneumothorax, or pulmonary embolism. TEE is especially helpful in the management of disorders such as acute pulmonary hypertensive crisis, intracardiac clot formation, and hypertrophic cardiomyopathy. ${ }^{4}$ The initial fear of rupturing esophageal varices is an exceedingly uncommon complication and has not yet been reported despite widespread intra-operative use. ${ }^{5}$

We would like to raise four points: (1) baseline TEE for OLT should seek to specifically identify trans-pulmonary shunts; (2) liver transplant candidates may be at increased risk of paradoxical embolism even if they do not have a PFO or severe hepatopulmonary syndrome; (3) one should note the presence of a TIPS that may contribute to life threatening air emboli during liver dissection; and (4) TEE is increasingly used in liver transplantation and can aid in the rapid diagnosis of a potentially underestimated issue of OLT air embolism.

Financial support None.

Conflicts of interest None declared. 


\section{References}

1. Mandell MS. Clinical controversies surrounding the diagnosis and treatment of hepatopulmonary syndrome. Minerva Anestesiol 2007; 73: 347-55.

2. Fung SK, Hui TW, Wong AK, Lei GM. Anaesthesia for liver transplantation: experience at a teaching hospital. Hong Kong Med J 1999; 5: 27-33.
3. Ozier $Y$, Klinck $J$. Anesthetic management of liver transplantation. Curr Opin Anaesthesiol 2008; 21: 391-400.

4. Krenn $C G$, De Wolf $A M$. Current approach to intraoperative monitoring in liver transplantation. Curr Opin Organ Transplant 2008; 13: 285-90.

5. Suriani RJ, Cutrone A, Feierman D, Konstadt S. Intraoperative transesophageal echocardiography during liver transplantation. J Cardiothorac Vasc Anesth 1996; 10: 699-707. 